\section{FREE RADICALS IN CHEMISTRY}

\section{The Chemistry of Free Radicals}

By Dr. W. A. Waters. Second edition. Pp. viii +296. (Oxford: Clarendon Press; London: Oxford University Press, 1948.) 20s, net.

$\mathrm{D}^{\mathrm{u}}$ URING recent years it has become increasingly evident that free atoms and radicals, chemically very reactive but physically quite stable, play an important part as intermediates in chemical reactions. Thus the equation of a chemical reaction, as usually formulated, is very often merely the sum of many separate equations involving their short-lived sub. stances, and a knowledge of their properties and reactivity is necessary if we are to understand the mechanism of the reaction as a whole. Consequently there has arisen in the last two decades a new kind of chemistry, no longer bound by the usual valency rules-the chemistry of free radicals. Dr. Waters' book surveys in a general way the whole of this rapidly growing subject.

The second edition, which has just been published, is identical in subject-matter with the first, published two years ago, but several minor alterations have been made and references to work published in 1947 are included. The approach to the subject is that of an organic chemist interested in understanding better the well-known reactions of organic synthesis. Physical methods of investigation, such as spectroscopy and mass spectroscopy and the results of theoretical chemists on free radical structure, are therefore omitted, although there is a fairly detailed chapter on the use of magnetic properties of free radicals as a means of diagnosis. After a general and historical introduction on the methods of production and detection, there are chapters on resonance-stabilized radicals of the triphenylmethyl type, the gas reactions of free atoms and the reactions of alkyl and aryl radicals and radicals containing other elements than carbon and hydrogen. These labile molecules are seen to be of common occurrence in a wide variety of chemical reactions. There follows an account of photochemical decompositions and the free radical mechanism of polymerization and oxidation. Finally, the subject is carried into the field of biochemistry, and the free radical mechanisms of biological oxidation are critically discussed.

The book is complementary in many ways to Dr. E. W. R. Steacie's "Atomic and Free Radical Reactions", first published at about the same time. Dr. Steacie is particularly concerned with the kinetics of the reactions of the simple free radicals mostly in the gas phase. and gives a very detailed and compre. hensive account of these reactions and their associated activation energies in so far as they are known. Dr. Waters, on the other hand, presents a more general and very readable account of a wider field and devotes more attention to reactions in solution. Each author is therefore writing particularly about that part of the subject to which his own research has contributed much.

Apart from the value to research workers in this relatively new branch of chemistry, Dr. Waters' book will be read with interest by a much wider public who want a general and lucid account of a subject which has applications in so many other branches of chemistry and biochemistry. In particular it will be of great interest to organic chemists in presenting a means of understanding better what are now almost classical methods of organic synthesis and preparation.
The Oxford University Press is to be congratulated on the production of this volume at the sort of price which is becoming rare in scientific literature. It is unfortunate that there is no term in the publishers' vocabulary with a meaning between 'new edition' and 'reprint' or 'new impression', for volumes of this kind which, though technically second editions, are not sufficiently changed to make their purchase necessary by owners of the first. In the absence of such a distinction a second preface or publishers' cover note indicating what changes have been made would be of value.

George Porter

\section{STELLAR ASTRONOMY}

\section{An Outline of Stellar Astronomy}

By Peter Doig. Second edition, completely revised. Pp. vii + 168. (London: Hutchinson's Scientific and Technical Publications, 1947.) 10s. 6d. net.

$T$ HIS little book is designed, as the author unassumingly states, for readers who have "slightly more knowledge than that necessary for perusal of 'popular' astronomical literature". It is, in fact, a useful summary, remarkably complete for its size, of the present state of knowledge, and there will be many genuine astronomers, both amateur and professional, who will find it worth reading for the facts and opinions which it reports.

It is not, however, a text-book, and although it does not claim to be one it must be said that it would have been even more useful if it had approached the text-book ideal more closely in some respects. In particular, although the facts, and the conclusions from arguments, are clearly stated, the arguments themselves are frequently so much compressed that they lack clarity; no one, for example, who is not already familiar with Trumpler's work on open clusters and interstellar absorption will be likely to grasp the argument summarized on p. 28 . In some places compression has resulted in actual errors; thus it is stated that the $v$-component of proper motion is caused by the sun's motion, and in the formulæ for mean parallaxes based on the $v$ and $\tau$ components no mention is made of the fact that with the former the means are algebraical while with the latter they are arithmetical; also the sign of $v$ is not defined, but appears to be opposite to the usual convention. These two are almost the only formulæ presented in the body of the book, and they are not actually usable as presented.

There are a number of good appendixes, mostly devoted to formulæ; one on indirect methods of parallax determination omits all notice of the three methods dealt with in the book proper, which may mislead some readers. There is a short bibliography at the end of each chapter, but it is quite clear that much more extensive and detailed references could easily have been given; they obviously were used by the author, especially in the appendixes (where scarcely any are quoted), and they would also have been valuable for elucidating the arguments in the text. There are a fair number of printer's errors, but most of these are minor. In spite of such criticisms the book well deserves a place on the shelves of working astronomers, in addition to having obvious value for the readers to whom it is more specifically addressed.
R. D'E. ATKRNSON 